\title{
BIG BEAR SOLAR OBSERVATORY
}

(NASA-CR-176571) THE TNO TYPES OF FLARE ASSOCIATED FIIAMENT ERUFTIONS (CaIifornia Inst. of Tech.) 23 p HC AO2/MF $\mathrm{AO} 12$

CSCL $03 B$

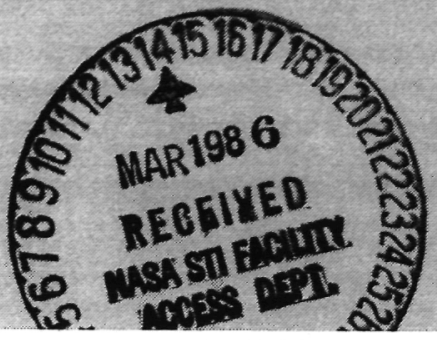

N86-21486

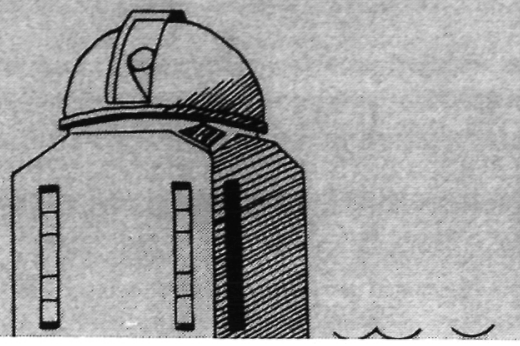

CSCL 03 B

\section{G3/92 05598}

THE TWO TYPES OF

FLARE ASSOCIATED FILAMENT ERUPTIONS

Frances Tang

BBSO \# 0255

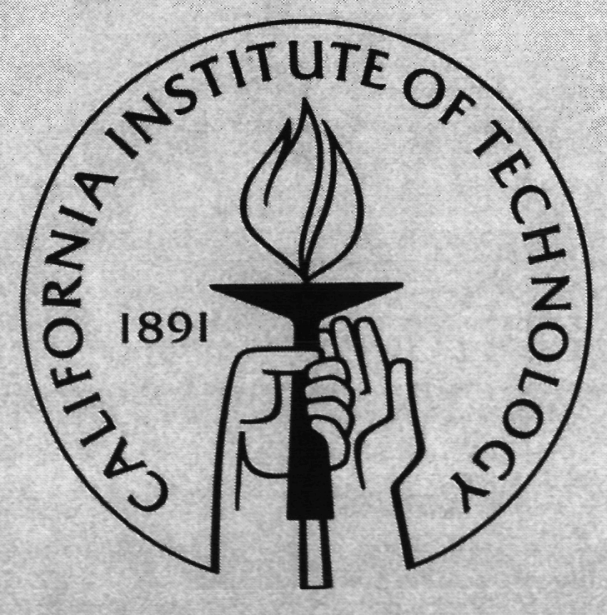

CALTECH ASTROPHYSICS PREPRINT 
THE TWO TYPES OF

FLARE ASSOCIATED FILAMENT ERUPTIONS

Frances Tang

BBSO \# 0255

January, 1986 


\section{ABSTRACT}

Using years of high resolution solar footage obtained at Big Bear Solar Observatory we studied flare associated filament eruptions. In addition to the "classical" type eruption consisting of expansion and breakup, we find evidence of another type where a layer is "shed" from the filament and erupts while the inversion line filament below (or, what is left of it) remains in place. Both types of eruptions are presented in the paper. It is hoped that the new evidence will shed new light on the understanding of the role of filaments in flares.

\section{INTRODUCTION}

Flares and coronal mass ejections are closely related to filament eruptions. Filament eruptions in active regions are intimately related to solar flares (Martin, 1980), but not every such eruption results in a coronal mass ejection. On the other hand, the eruptions of quiescent filaments (prominences) which may not always have an associated chromospheric flare are intimately related to coronal mass ejections (Munro et al., 1979). It is as though the eruptions of active region filaments may or may not affect a large part of the corona above them, whereas the eruptions of quiescent filament, being at much greater heights than active region filaments to begin with, disturb a greater part of the solar atmosphere above.

An understanding of the physical process of filament eruptions is crucial to the understanding of the magnetic topology in and around the filament, which, as Pneuman (1983) pointed out may well be one of the central problems in the whole solar activities area.

Filaments are familiar $\mathrm{H} \alpha$ absorption features of the chromosphere and corona. They mark the inversion line of the photospheric magnetic fields. Although many filament eruptions precede the onsets of flares, it is by no means a rule. Filament eruptions are also observed to take place simultaneously with flares, during flares, or as a result of the spread of the flares. Some filaments are observed to remain in place throughout the flare. In other cases flares are observed to take place without an inversion line filament. The 
diverse behaviors of the flare-associated filament eruptions raise the inevitable question of the role of the filament and its eruption in the flare scenario. However, the problem cannot be meaningfully addressed without an understanding of the physical process of filament eruptions. To this end, better observational understanding of the physical process of eruptions is crucial.

Flare associated filament eruptions had been studied for nearly four decades on low resolution data by many investigators. For summary, see the review by Martin (1980) and references therein. Essentially, the eruption process was observed to consist of the extension of a filament in the shape of a loop, the breaking up of the loop at the top, and the blowing off and disappearance of the flament material. This basic picture remains largely unchanged even with the advent of high resolution data as well as periods of space borne observations during Skylab and Solar Maximum Mission.

Although flare-associated filament eruptions are common occurrences, good and revealing observations are hard to come by despite years of high resolution optical data. This is because filament eruptions are difficult to observe even under the best circumstances. They happen quickly and are often obstructed by flaring material along the line of sight. Most filament eruption observations offer no more useful details than the basic picture described earlier; some are too complex to understand. However, a number of cases, collected from 15 years of high resolution solar footage at Big Bear Solar Observatory, offer more information on the eruption process. The high resolution data reveal a new type of eruption where a layer is shed or peeled off from the active region inversion line (IL) filament. This layer then erupts while the $I L$ filament below remains in place. The events of this type are presented in section 2. For comparison, the "classical" type of eruption is presented in section 3. Summary and discussion are offered in the final section.

\section{PEELING AND ERUPTION OF A FILAMENT LAYER - A DIFFERENT TYPE OF FILAMENT ERUPTION}

\subsection{An Overview}

This is the type in which the inversion line filament remains in place but a layer of 
it is seen to separate from the parent filament. The severed layer is displaced from the parent II filament such that it is at a higher altitude and generally does not lie directly above the photospheric magnetic inversion line.

This transient layer may erupt as it peels off the parent filament, or it may stay there for tens of minutes before erupting. The manners in which the layer erupt vary. In one case, a loop-like layer took off as a unit. In most cases, however, the mass in the layer seems to move along the expanding partially relaxed field lines. Often chromospheric brightenings may be seen at one end or both ends of the field lines when part of the mass falls downward toward the surface.

Of the 15 events we collected where the observations offer details of the eruption process, eight fall into the 'eruption of a filament layer' catagory. The rest belongs to the "classical" type of eruption. Because of the selection criteria, the numbers bear no statistical significance.

\section{$2.2 \quad$ Examples}

a). April 26, 1979

In this event shown in Figure 1, the separation of a layer from the inversion line filament was evident at 195522 UT. A two-ribbon flare began at 1958 UT. The IL filament, seen here in +.5 Aat 200320 UT, remained in place throughout the flare, even though it was not as visible in the line center until the two flare ribbons had receded sufficiently enough from the inversion line. The loop-shaped top layer, seen here in $2 \AA$ blue wing at 200314 $\mathrm{UT}$, in the mean time retained its cohesion and sailed away as a unit until it disappeared outside of the field of view at 2010. Interestingly no associated coronal mass ejection for this event was observed despite adequate Solwind coronagraph coverage (Sheeley, 1985, private communication).

b). June 22,1980

This is a very small region and the event was the second one of a similar kind on the 
same day. Figure 2 shows that a layer of the $\mathrm{IL}$ filament rose and moved across to the right to a less sheared orientation. At 2011, a small but implusive flare began and the layer blew off in the upper left direction. A small burst with maximum at 201415 was observed by the SMM Hard X-Ray Burst Spectrometer.

c). Oct. 2, 1979

This sequence in Figure 3 shows a layer of a filament came off as a result of a flare nearby. Unlike the others, in this case the eruption of the severed layer did not result in a flare.

d). Oct. 9,1977

The sequence in Figure 4 shows how a segment of the top layer separated from the IL filament as the flare began. The layer then "caught fire" (155632), became completely detached (160021) and then drifted away. Note again that the IL filament remained in place throughout the flare. Three hours later, another segment of the IL filament thickened and at 192913 UT the top layer of that segment became detached and erupted, accompanied by a very small flare.

e). May 16, 1983

This event shown in Figure 5 consisted of two flares, or two stages of one flare. The top layer appeared as a hump at 152923, and became separated from the IL filament as a result of the first flare. The elevated layer seemed to indicate a less sheared field in the 162412 frame than that of the IL filament below. This layer then erupted with a second flare (163612), leaving the IL filament intact below (175857).

f). June 9,1979

This event (Figure 6) is very similar to the May 16, 1983 event just presented. They differ only in perspective due to different longitudes through which the observations were made. The first frame shows the flare on June 8 (2220-2236-2318) in progress. At 2339, a layer is seen being shed. The second flare, began at 2344 as the top layer lifted from the 
IL filament.

In this June 9 event, unlike the others, the transient layer did not erupt and disappear but began to descend at 0018 and completely merged with the IL filament by 0043 !

g). March 4, 1981

A detached and elevated layer of the filament (arrow) not seen at the end of the previous day at 2330 was there when observation began on this day at 0722 at the $\mathrm{Tel}$ Aviv station, Figure 7. We infer that the layer "grew" out of the IL filament just as in the other cases we witnessed. The transient layer began to erupt at 0725 , slowly in the first $80 \mathrm{~min}$ then speeding up. A class 1 flare began as the last trace of the detached layer disappeared.

\section{FILAMENT ERUPTION OF THE "CLASSICAL" TYPE}

In contrast to the eruption of a layer of filament discussed in the last section, in this "classical" type of eruption the entire filament takes part. The extension of the filament loop before breakup of the field lines is observed in this case. We present two large events of this kind because they offer exceptionally good observations that may prove valuable in the understanding of this type of eruption process. The events occurred at large longitudes so that they are viewed in their true three-dimensional form.

Figure 8 shows an eruption on October 10,1971 at N12 W73. The 1/M5 flare appeared to begin under the filament. As the bright blob expanded, the filament extended as though pushed from underneath. With both feet anchored, the filament expanded until strands of the field lines began to break open at the top at various heights. The highest loop visible reached about $100,000 \mathrm{~km}$. By 0100 UT no more closed loops were visible. The observation ended at 0104 with both feet of the filament still firmly anchored to the surface.

Figure 9 shows an event very similar to the one just discussed. This more impulsive 2/X1.9 flare was at N16 W65. Again the flare seemed to begin from under the filament. As the filament extended strands of the filament loops popped open at the top. Both feet of the filament were anchored to the surface as indicated by the arrows in the 005544 frame. 
The top of the visible loop is estimated at $250,000 \mathrm{~km}$. (At this point the telescope was repositioned and exposure was increased to enhance the over-the-limb visibility.) Mass was seen to continue to ascend upward. However, we are uncertain as to the origin of this mass since the geometry of the loop looked different. Nevertheless we find it worthwhile to report it here since it offers a rare chance to observe an eruption in three dimensions; we probably could not "decode" it corrrectly had it occurred on the disk. At $\sim 0100$ UT slanted loops appeared. Material was seen to move up one leg and down another along the individual loops (reminiscent of spiralling of the falling material in flare spray described by Tandberg-Hanssen et al., 1980). At the same time, the loops themselves are seen to descend back toward the surface (see 011940 frame). When the flare was over, the filament (seen in the first frame at 0007) was gone.

The two near-limb events just presented show that the feet of the filament are rooted in the solar surface during the eruption process and at least some mass is seen to return to the surface. It is not known whether the mass in the expanding phase of the eruption comes from the filament itself, i.e. with no net increase in mass, as in the case of a stretched rubber band; or, the mass comes from a "live" source in the lower atmosphere where the feet of the filament are rooted, as in the case of a surge. Except in surges only one foot is anchored to the surface.

\section{DISCUSSION}

We have shown that there are two ways filaments may erupt. We now discuss the implications and problems brought on by the new evidence.

a). Do Filaments Really Re-form During Flares?

In the eruption of a filament layer (section 2), a layer becomes detached from the $\mathrm{IL}$ filament. When this detached layer erupts, the $\mathrm{IL}$ filament below (or what is left of it) remains in place. Because of the obstruction of flare ribbons, this information is lost in full disk low resolution data. Instead, the observer sees the eruption of a filament and when the flare ribbons separate sufficiently from the magnetic inversion line, he sees "another" filament. This explains most, if not all, of the many reports on "reformation" 
or "reappearance" of filaments during flares in the literature.

\section{b). Large Flares Associated with Eruptions of a Filament Layer}

The eruptions of a layer of detached $\Pi$ filaments have been observed with a few large well-known flares, although they were not understood as such at the time. One such event involved a 6 arc minute long layer crisscrossing the IL filament at two points, whose eruption on September 4, 1982 resulted in a class 3 flare lasting 19 hours (see the Frontispiece Solar Phys. 83, 2; Harvey and Recely, 1984; Morishita, 1985). Arrows in Figure 10a show segments of that layer above the IL filament not yet joined together. Reexamination of our full disk footage reveals that the first segment of the layer began to rise out of the sinuous II filament at 2318UT. Other segments, elevated and inclined from the photospheric magnetic inversion line, appeared later and joined together to become 6 arc min long by 0032UT on September 4.

In another example, on July 29, 1973 (Martin, 1979; Moore and LaBonte, 1980) the eruption of a detached layer of filament appearing as a dark mass hanging 20,000 km over the IL filament, shown in Figure 10b, whose eruption resulted in the Skylab class 3 flare. We infer the layer was shed from its parent $I L$ filament below in the same way as the others between 0200 and 0710 , the data gap between our two sets of film footages.

\section{c). Evidence of Severed Filament Layer in Soft X-Rays}

In a recent comprehensive survey of Skylab soft X-ray images Webb (1985) reported a significant number of preflare X-ray enhancements in the shapes of loops, knots and sinuous features that are in general displaced from the flare sites. $\mathrm{H} \alpha$ absorption features were found in half of these cases. Some of these preflare X-ray enhancements could well be caused by the detached layer of filaments, since thermal X-ray enhancements were observed to coincide with preflare filament disruptions (Roy and Tang, 1975).

\section{d). What Does the New Evidence Imply?}

If filament eruption is triggered from underneath by instability in the field lines, then it may be that the two kinds of eruption are different only in the location of the triggering instability. If it is under the filament, the whole filament will erupt; if the instability is 
imbedded within the filament, only the top layer will erupt. However, this does not explain why and how a severed layer becomes suspended high above the inversion line filament stably for up to tens of minutes.

Does the presence of this elevated layer signify a topological change in the magnetic field at that height to a more sheared configuration? When the severed layer erupts and the inversion line filament remains underneath, does this imply the flare disturbance leaves the lower layer of the atmosphere unperturbed? These and other questions on the physics of the inversion line magnetic field, in view of the new findings presented here, cannot be answered without a thorough theoretical consideration which is beyond the scope of this paper.

We may not have captured all facets of filament eruptions at present. As the level of understanding increases, future observations will undoubtedly unravel more. Nevertheless, we hope the new evidence unveiled here will inspire a new generation of models for theoretical consideration and interpretation of the magnetic topology in and around the filament and its role in the flare scenario.

ACKNOWLEDGEMENTS: I thank H. Zirin, S. Martin and D. Gary for helpful discussions. This work is supported by NASA Grant NGL 05002034 and NSF ATM 8513577. 


\section{REFERENCES}

Harvey, K.L. and Recely, F. 1984, Sol. Phys. 91, 127

Martin, S. F. $\quad$ 1979, Sol. Phys. 64, 165

Martin, S. F. and Ramsey, H. E. 1972, Solar Activity, Observations, and Predictions, MIT Press, Cambridge, Mass. Vol. 30, 371

Morishita, Hirozo 1985, Tokyo Astron. Bulletin, No. 272, 3123

Moore, R. L. and LaBonte, B. J, 1980, Solar and Interplanetary Dynamics, M.Dryer and E. Tandberg-Hanssen eds., 207

Munro, R. H., Gosling, J. T., Hilder, E., MacQeen, R. M.,Poland, A.I. and Ross, C. L., 1979, Sol. Phys. 61, 201

Roy, J.R. and Tang, F. 1975, Sol. Phys. 42, 425

Tandberg-Hanssen, E.; Martin, S. F. and Hansen, R. T. 1980, Sol. Phys. 65, 357

Webb, D. F. 1985, Sol. Phys. 97, 321 


\section{FIGURE CAPTIONS}

Figure 1: A layer of the inversion line filament is seen to separate from the parent filament. The loop shaped layer sailed away as the class 1/C7 flare began at N11 E31. The IL filament remained in place throughout the flare. A metric Type II burst was observed 2015-2035 UT. But no coronal mass ejection was observed despite adequate coverage.

Figure 2: A layer of the IL filament rose and separated from it. The eruption of this layer touched off an implusive subflare 2005-2014-2032, located at S23 E14.

Figure 3: This sequence shows a layer of the filament peeled off and erupted with no associated flare except a single blob of brightening.

Figure 4: This sequence shows how the top layer of a segment of the parent filament broke apart from it, then erupted. The associated subflare was located at N13 E36. Note that three hours later (last frame) another layer became detached and erupted with a small flare.

Figure 5: The top layer not yet separated from the parent filament appeared as a hump at 152923 UT. The separation was complete as a result of a subflare at 162412 UT. Note that the layer seemed to be in a less sheared configuration than the parent IL filament below. A class 0/C5 subflare at S29 W54 accompanied the eruption of this layer.

Figure 6: Viewed at a different perspective, this event is the equivalent two-dimensional version of the one in Figure 5. The associated 1/M1 flare was located at N23 W32. Unlike the others, however, in this event the severed layer did not erupt upward and disappear but descended and merged with the parent filament as shown in the last frame.

Figure 7: Arrow indicates the layer already separated from the IL filament when the observation began. This class 1 flare (Peak soft X-ray intensity is not known due to data gap) began at 0923, after the eruption of the detached layer completed. We infer the layer originated from the parent $\mathrm{IL}$ filament in the same way as in the other cases. The flare at S17 W36 lasted until 1230 UT with maximum at 0946 UT.

Figure 8: At W73, this filament eruption/flare illustrates the "classical" type of eruption where the filament expanded, followed by breakup and disappearance. Note that the feet of the filament remained anchored to the solar surface throughout the expansion and breakup. Note also that the flare began under the filament. The filament extended as if pushed from underneath. A $\sim \mathrm{M} 5$ in maximum soft X-ray flux was measured by OSO-7. 
Figure 9: This June 26, 1982 event is very similar to the Oct. 10, 1971 event. The arrows (005544 UT) indicate the feet of the filament. See text for detail. When the flare ended, the filament (seen at $000750 \mathrm{UT}$ ) was gone. The implusive $2 / \mathrm{X} 1.9$ flare was located at N16 W65. Two hard X-ray bursts were recorded, the second was accompanied by a radio burst up to $35 \mathrm{GHz}$.

Figure 10: Two class 3 flares associated with eruptions of a filament layer. In frame a), arrows show segments in the early stage of an elevated layer above the inversion line filament that eventually grew to be 6 arc min long on September 4, 1982. In frame b), the arrow indicates the massive layer hanging over the IL filament before its eruption and the class 3 flare on July 29, 1973. 


\section{Cinnmen bace to \\ Cr roon crimtiry}

April 26. 1979

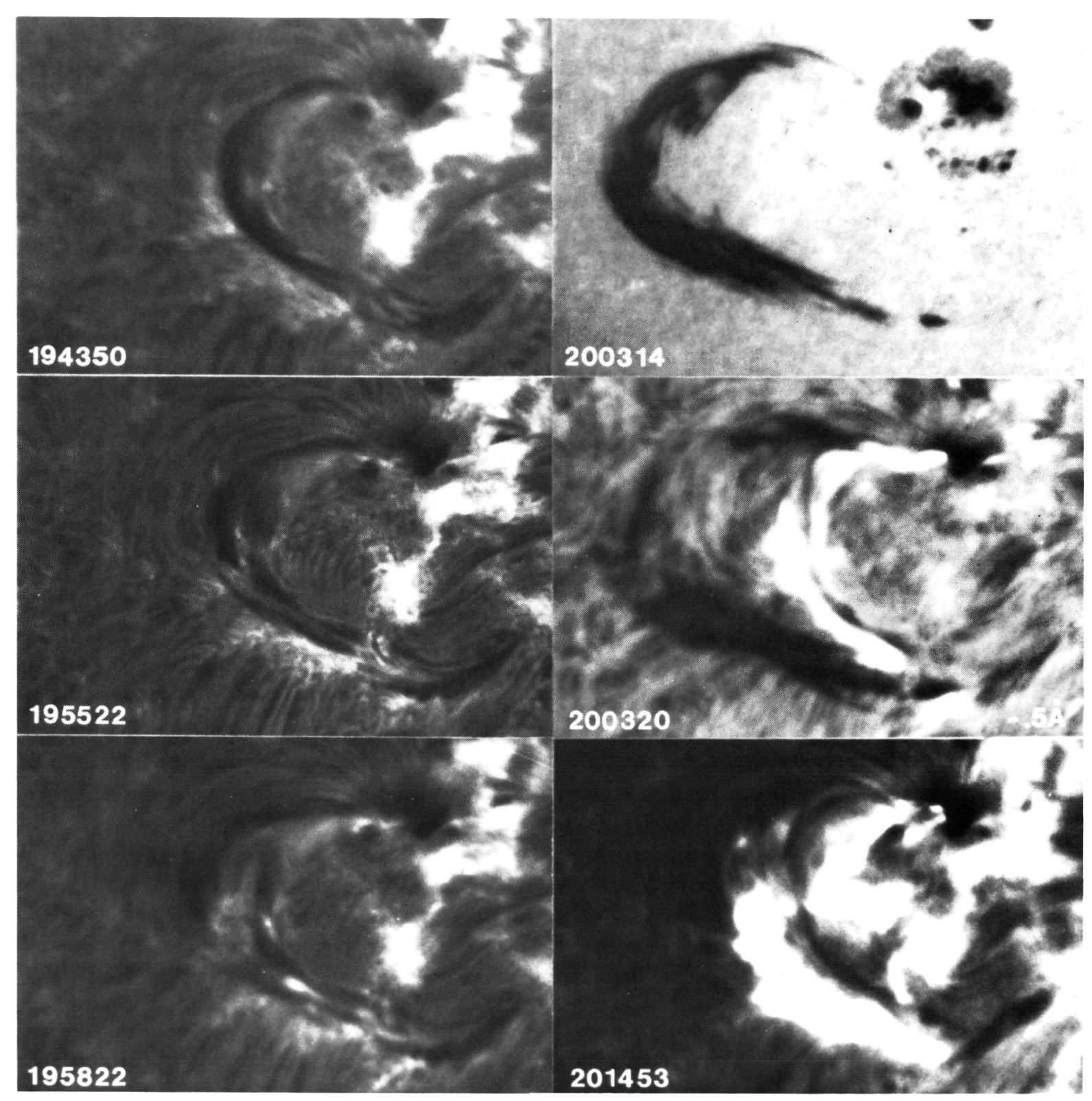




\section{ORIGINAL PAGE IS}

OF POOR OUALTTY

$6 / 22 / 1980$

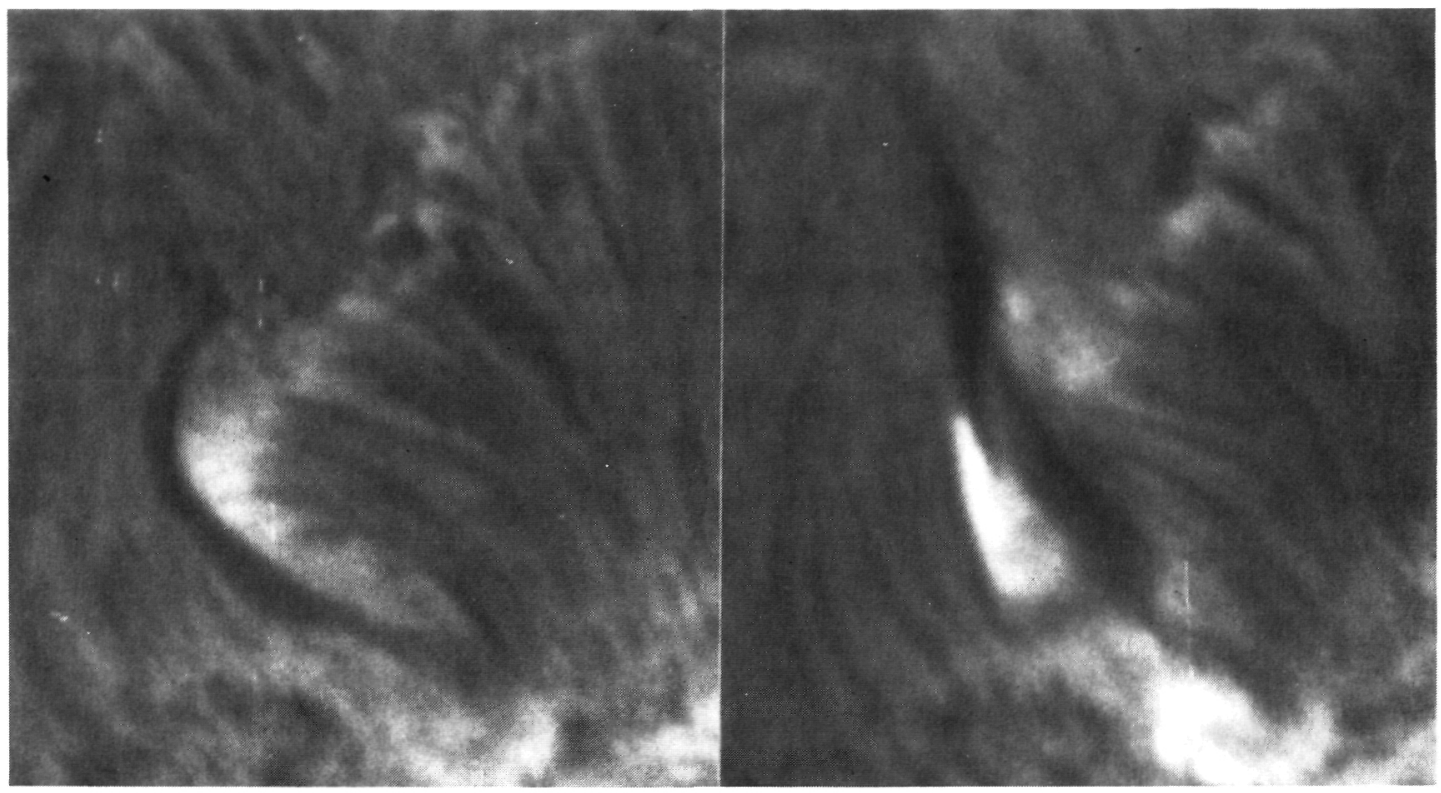

194834

201121

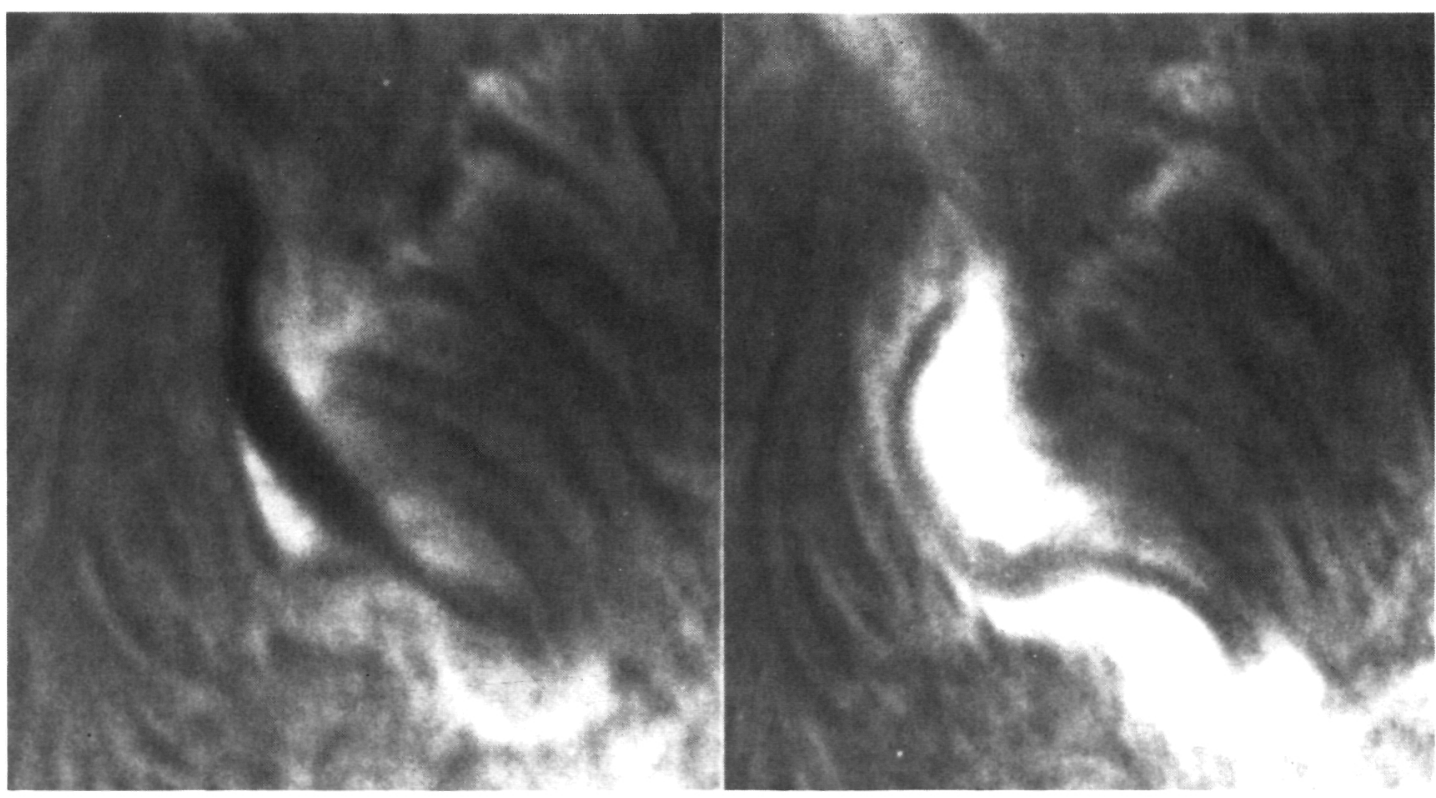

200521

201621

FIGURE 2 


\section{$10 / 2 / 1979$}

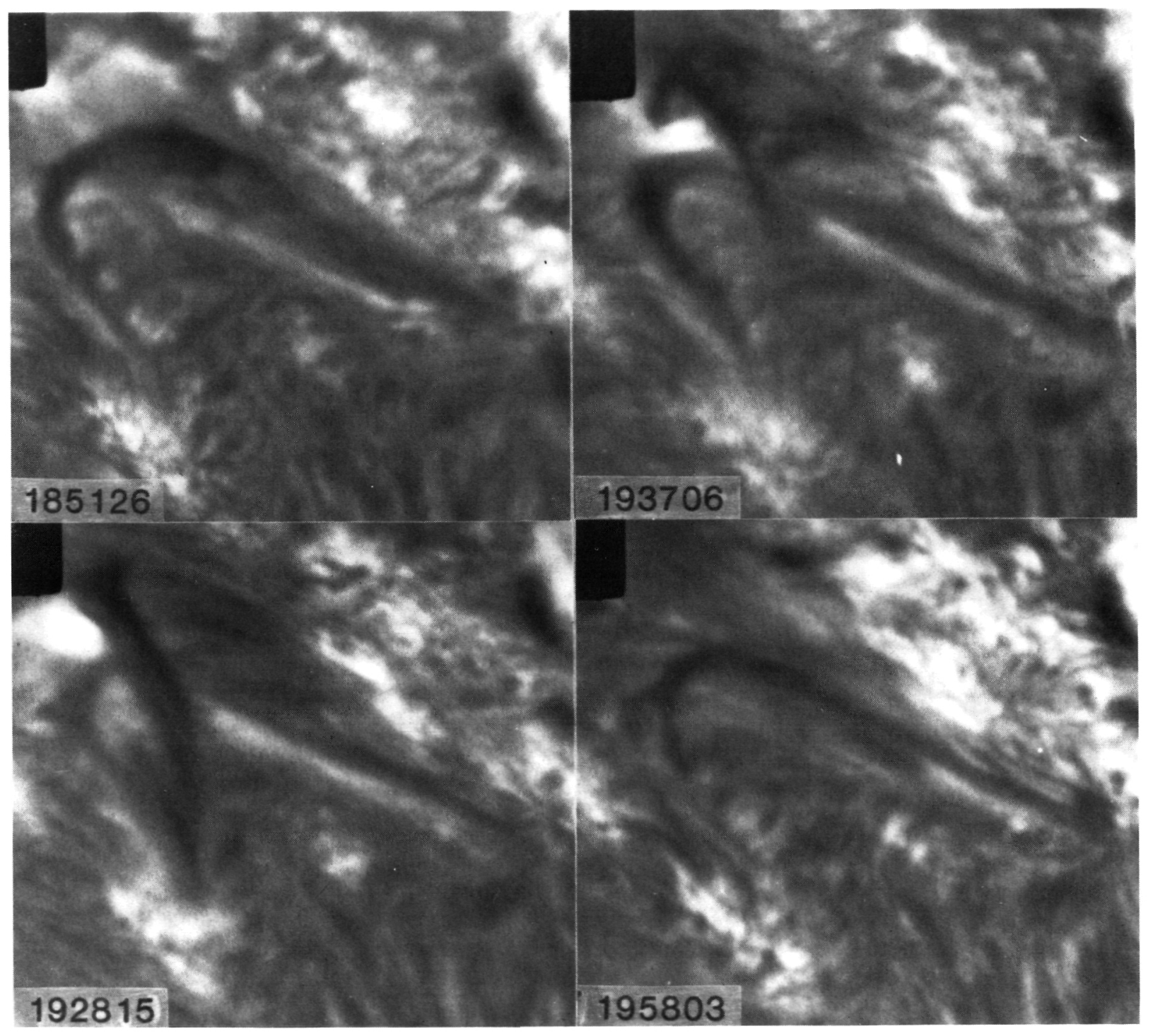

FIGURE 3 


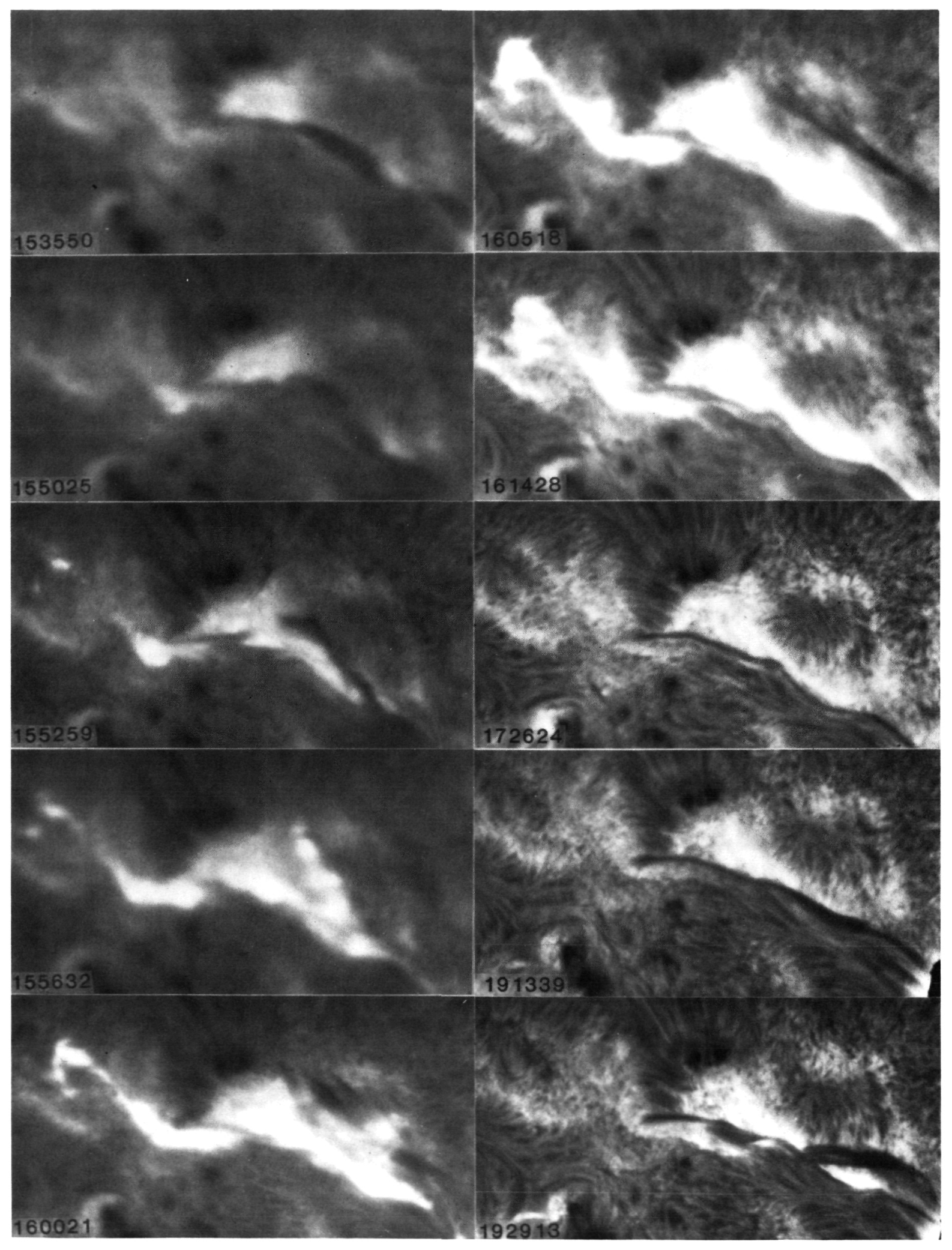

FIGURE 4 


\section{$5 / 16 / 1983$}

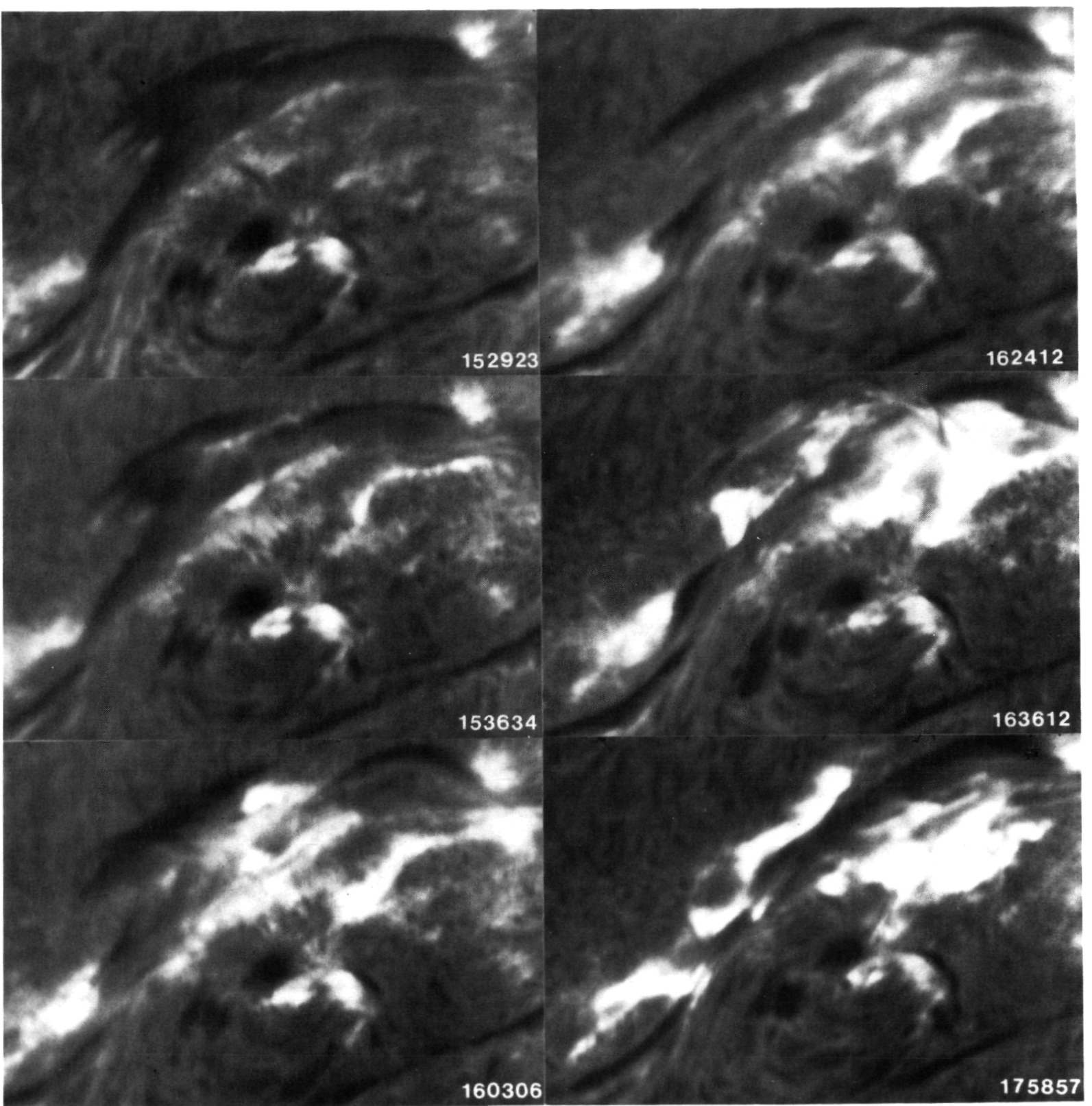

FIGURE 5 


\section{ORIGINAL PAGE IS \\ OF PONR OUJALTY}

\section{$6 / 9 / 1979$}

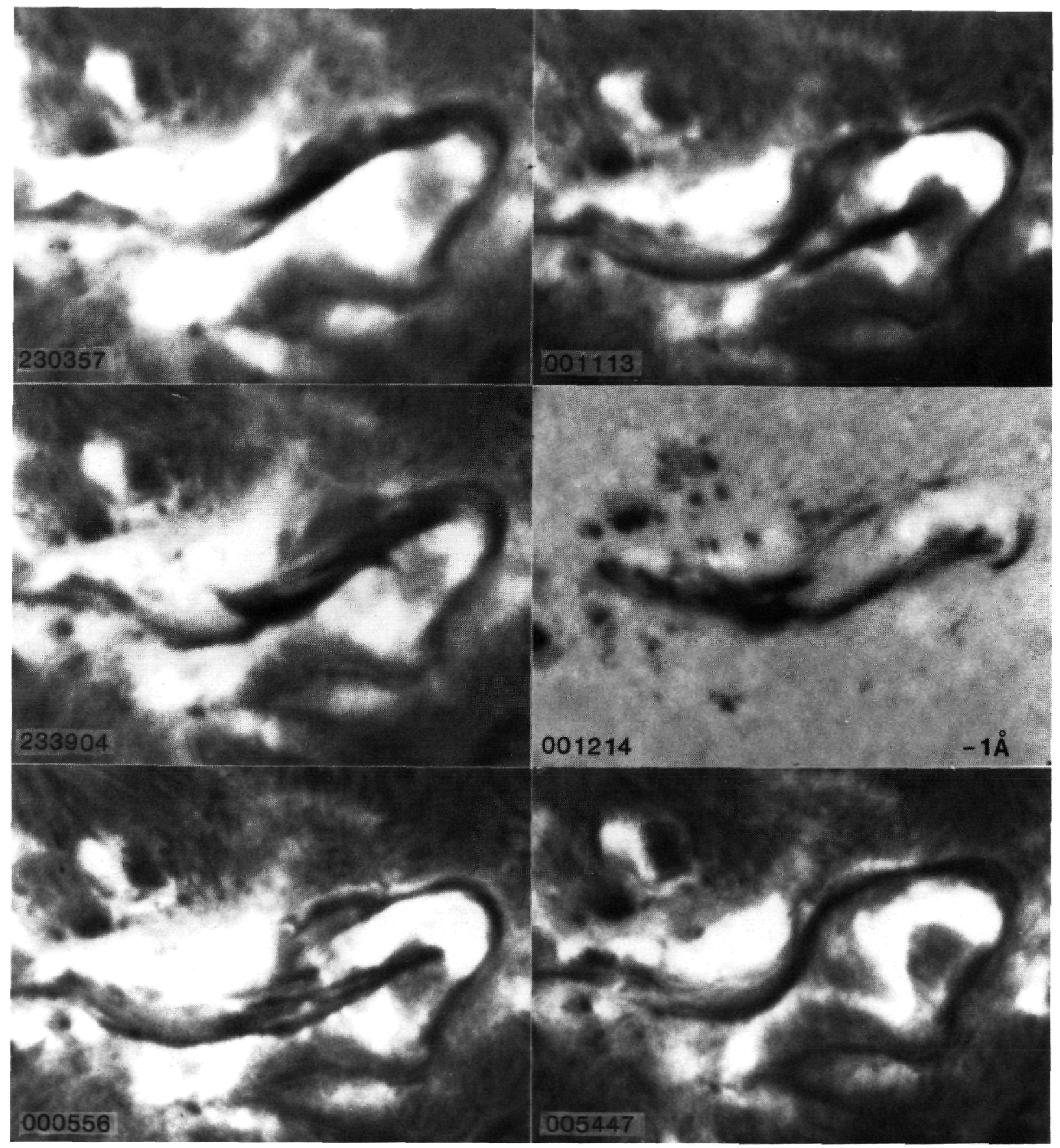

FIGURE 6 
ORIGINAL PAGE IS
OF POOR QUALTTY

\section{$3 / 4 / 1981$}

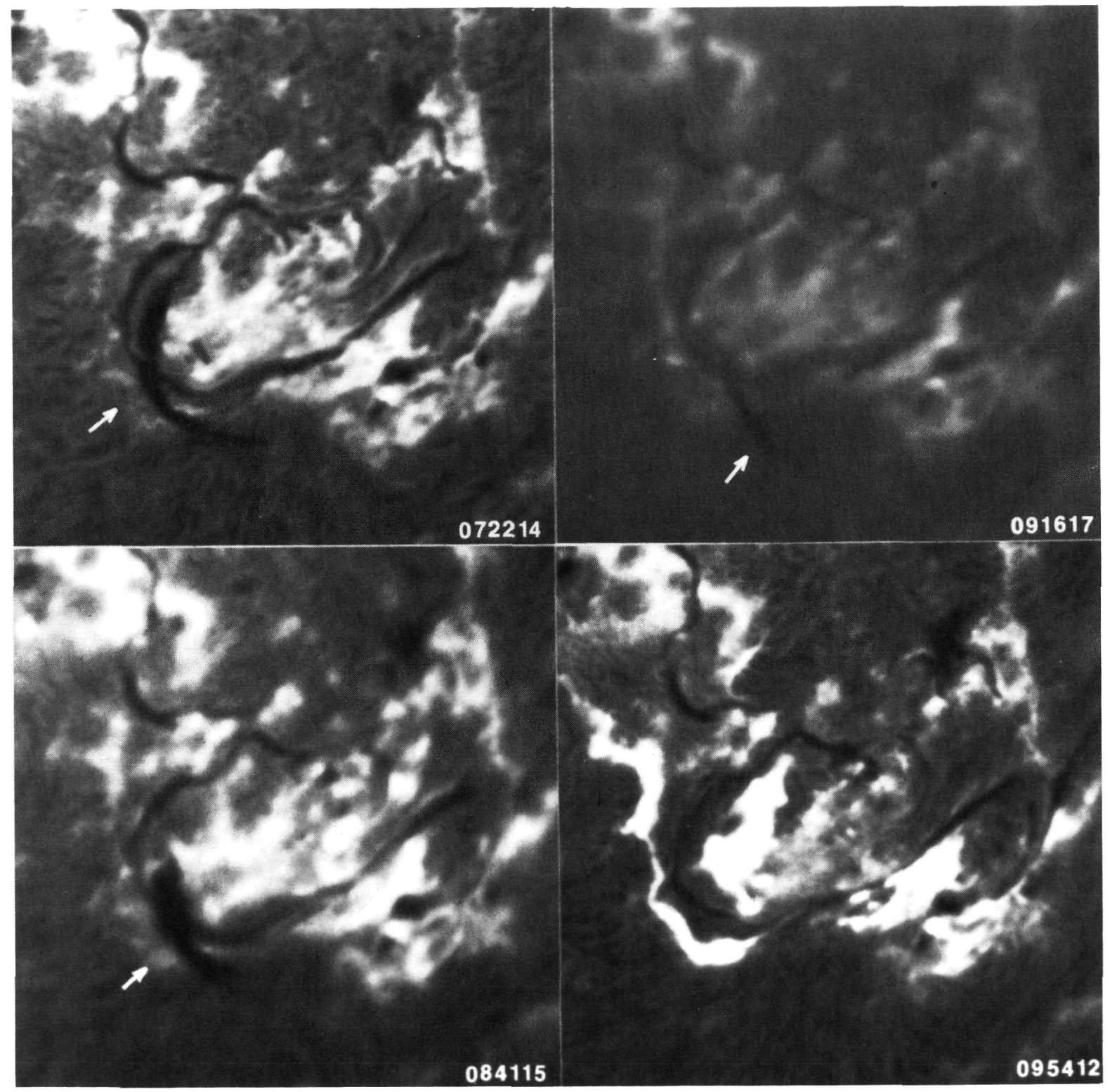

FIGURE 7 


\section{Covenua page is
cf poor pualty}

OCTOBER IO, 197|
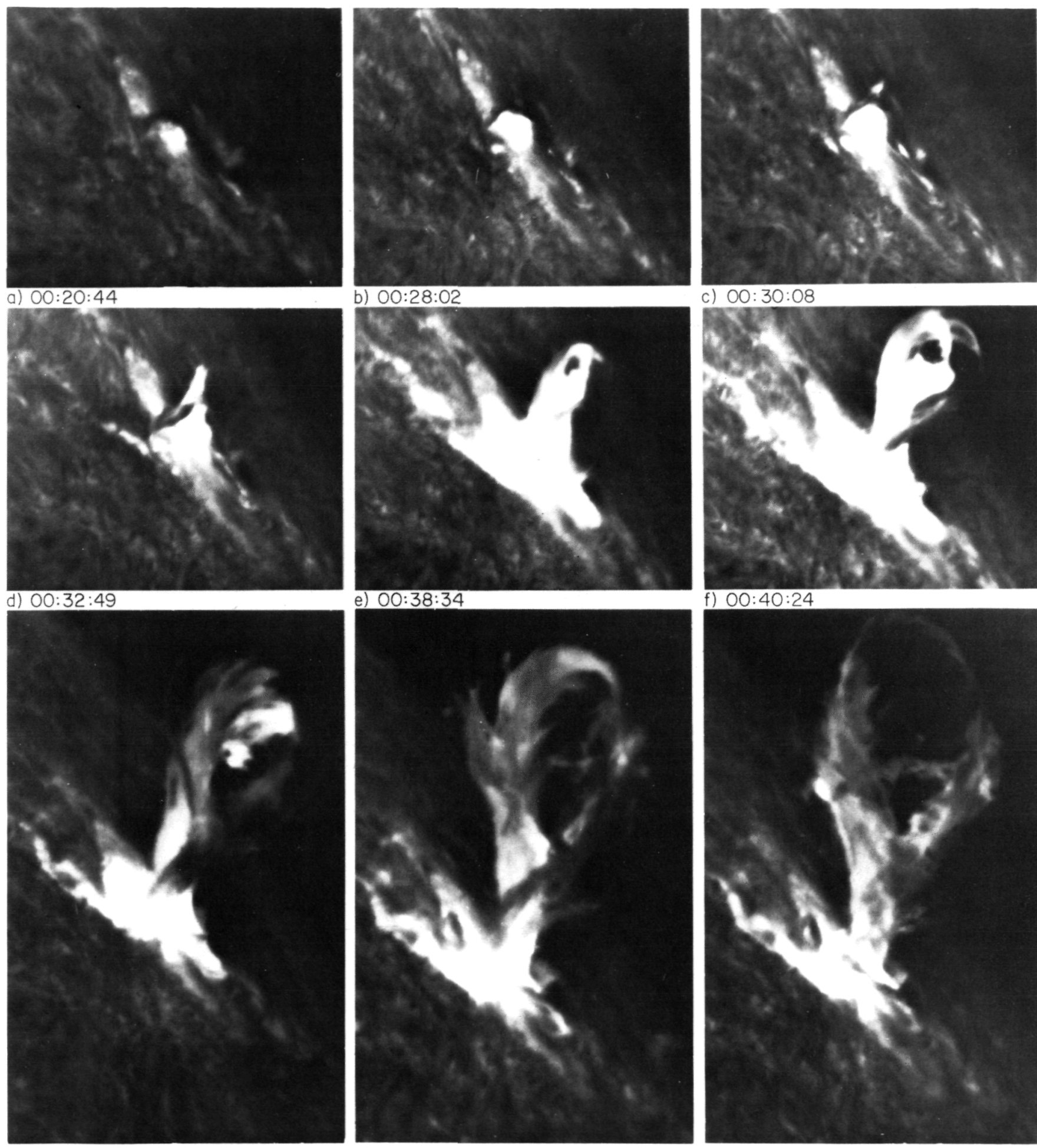

g) $00: 44: 32$

h) $00: 51: 46$

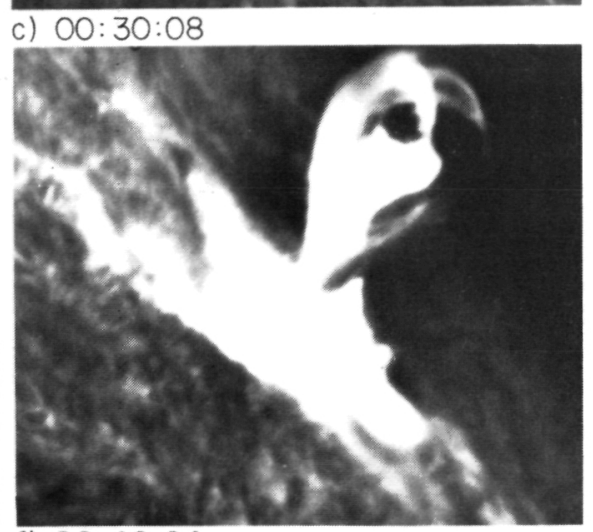

f) $00: 40: 24$

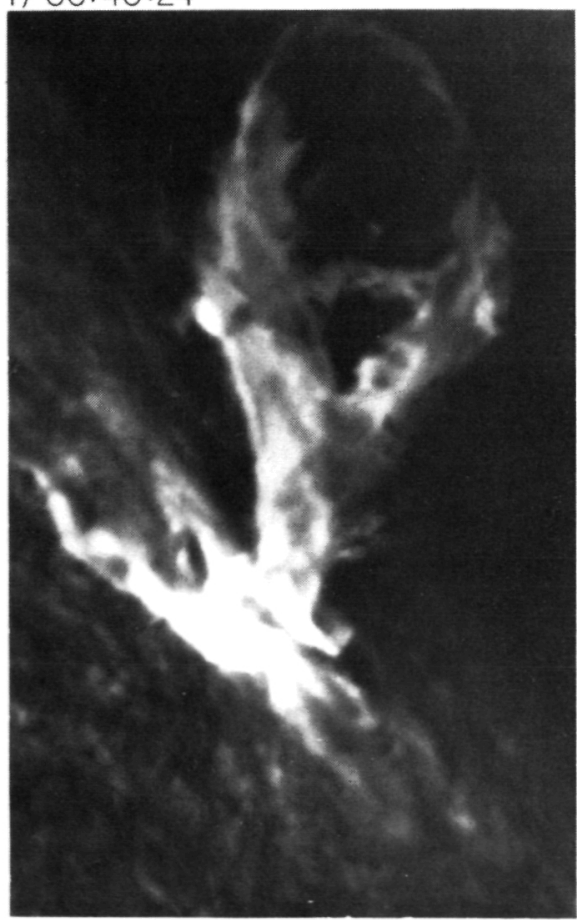

i) $00: 55: 11$

FIGURE 8 


\section{ORIGINAL PAGE IS OF POOR QUALITY}

\section{$6 / 26 / 1982$}
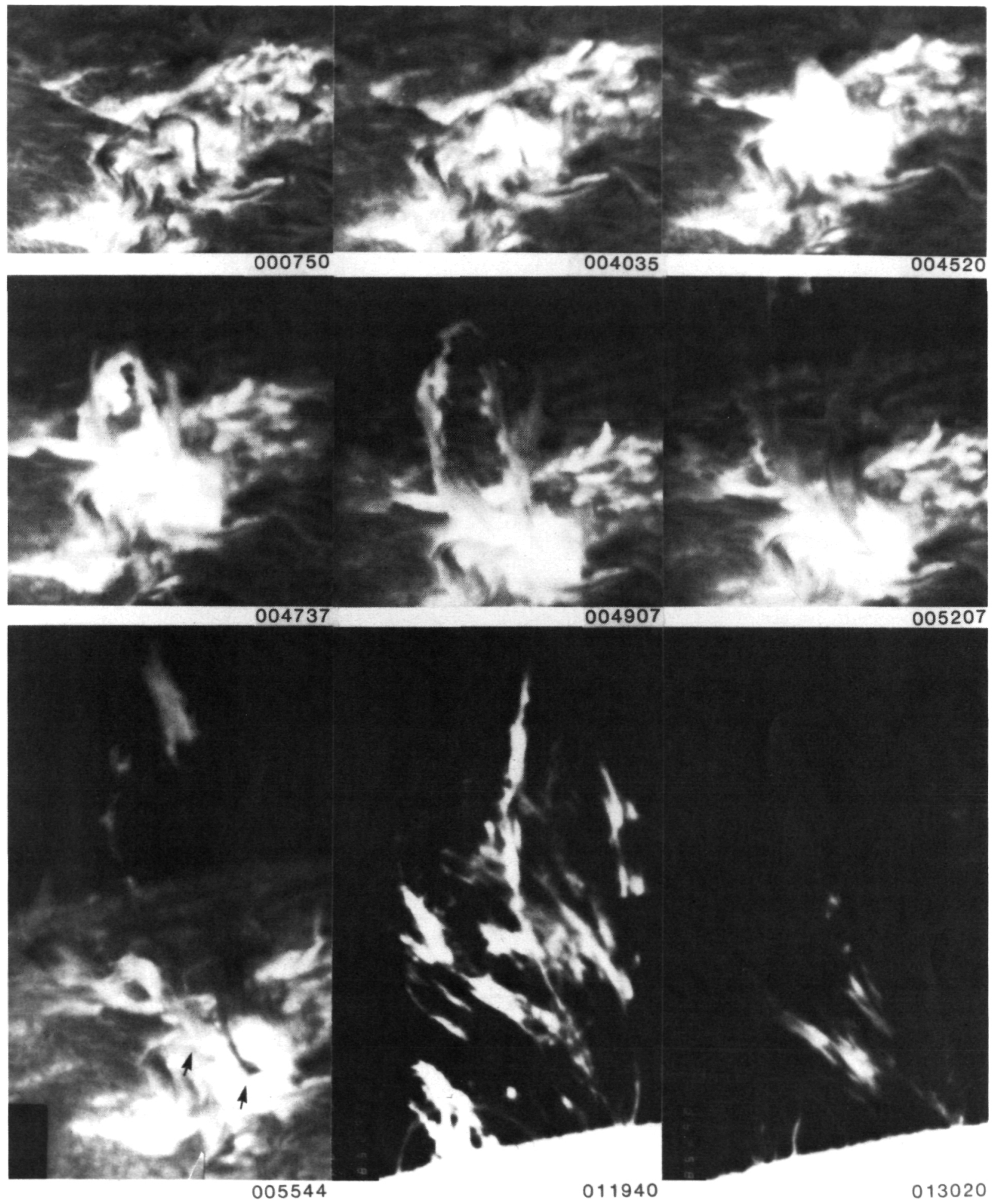

FIGURE 9 
oivemal PAGE IS

$9 / 3 / 82$

OF POOR QUALTYY

a

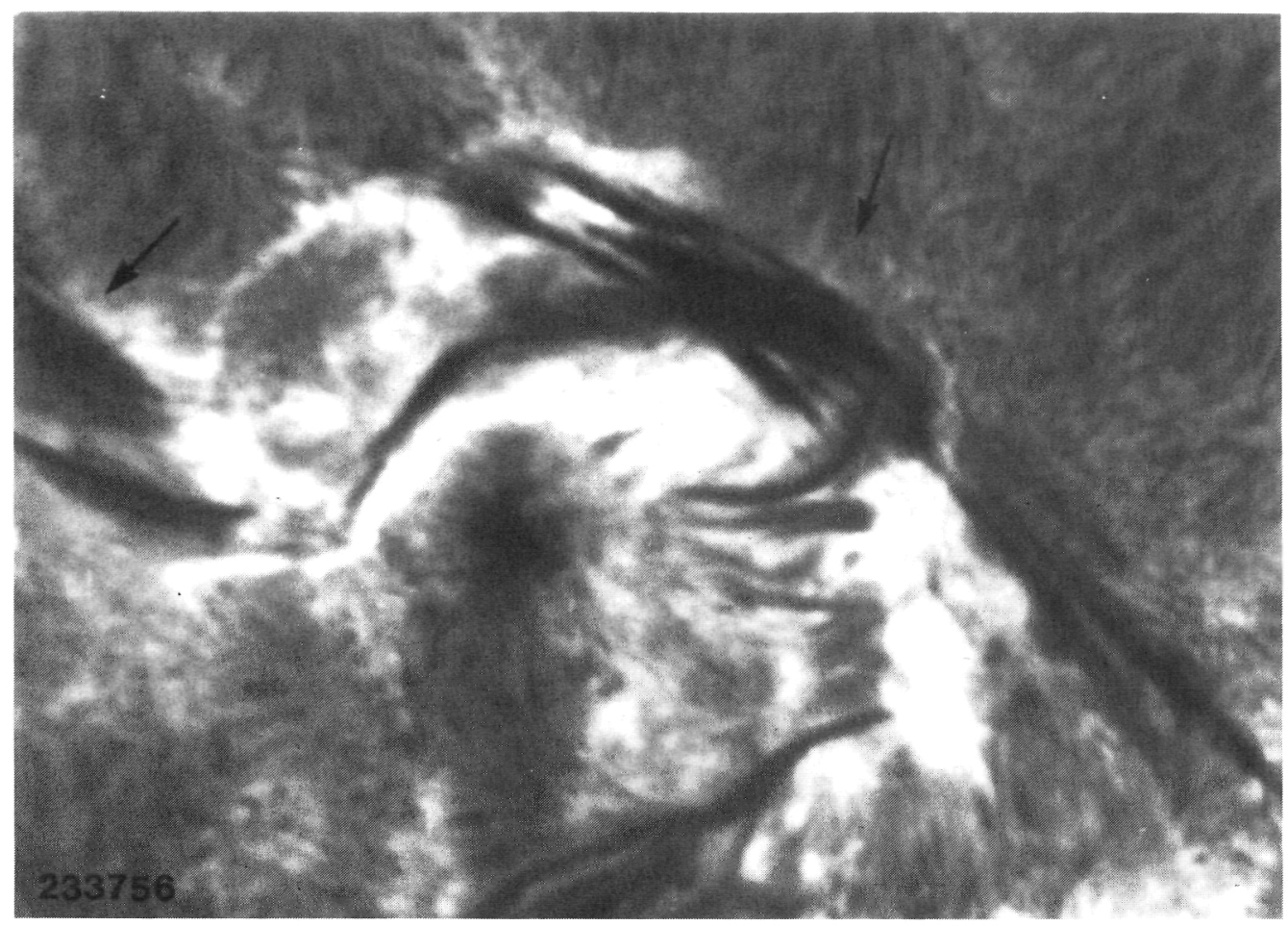

\section{$7 / 29 / 73$}

b

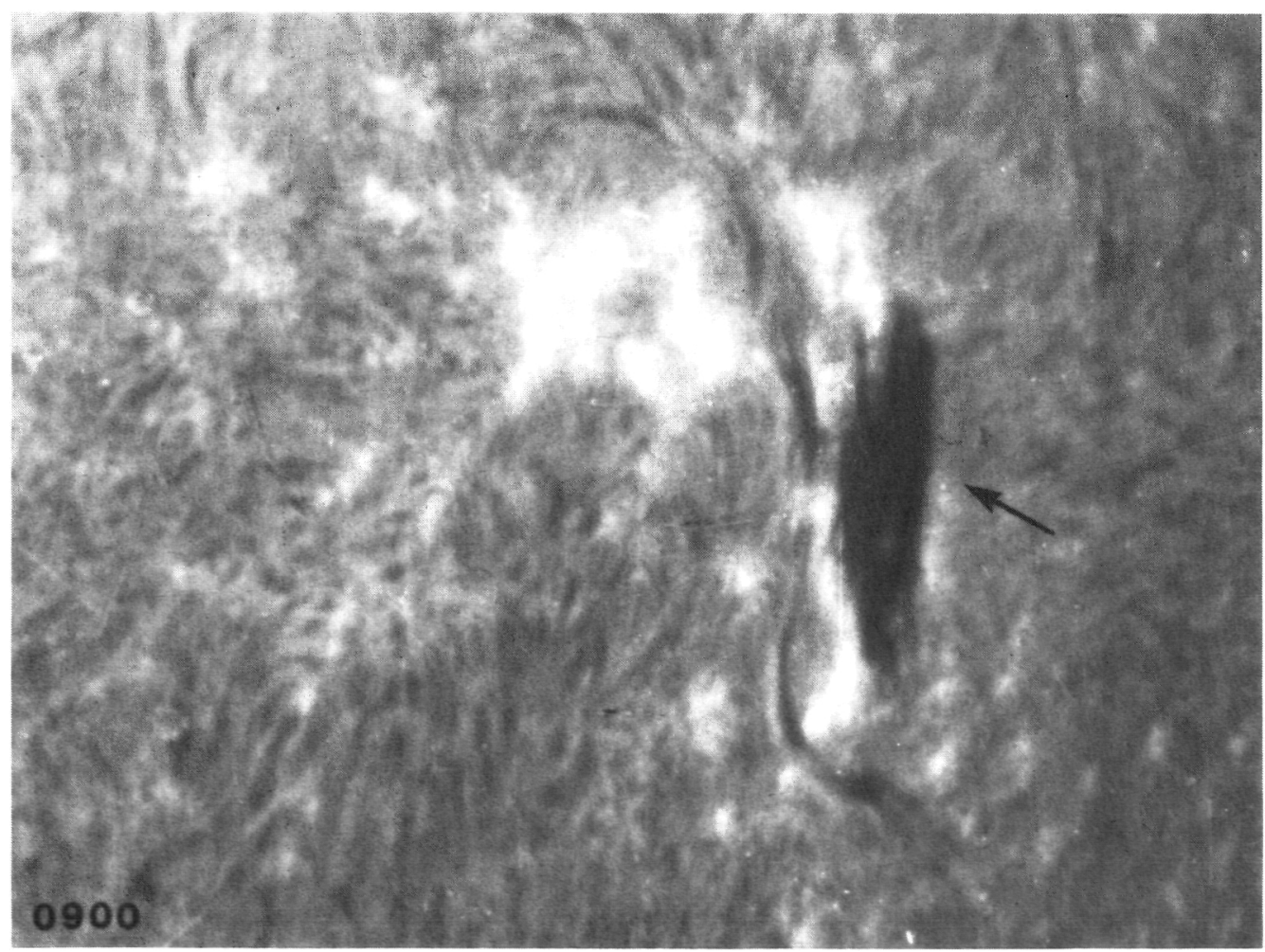

FIGURE 10 


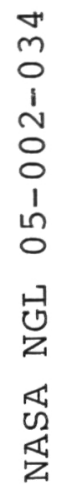

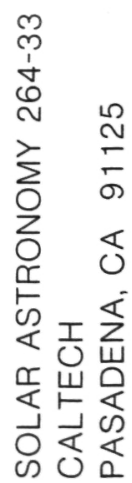

